\title{
Triple Therapy for Persistent Pulmonary Hypertension Post Left Ventricular Assist Device: A Success Case for Cardiac Transplant
}

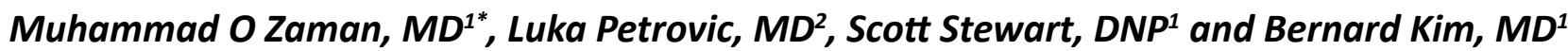 \\ ${ }^{1}$ Hackensack University Medical Center, Hackensack, NJ, USA \\ ${ }^{2}$ Rutgers New Jersey Medical School, NJ, USA \\ *Corresponding author: Muhammad Omer Zaman, MD, Department of Advanced Heart Failure and \\ Pulmonary Hypertension, Hackensack University Medical Center, 20 Prospect Ave, Suite 201, Hackensack, \\ NJ 07601, USA, Tel: 424-298-1685
}

\begin{abstract}
Patients with end stage heart failure often develop pulmonary hypertension which resolves after placement of left ventricular assist device (LVAD). Some patients develop persistent pulmonary hypertension despite LVAD implantation. This excludes them from being able to receive a heart transplant. High pulmonary vascular resistance is associated with poor prognosis in patients with LVAD. There are no treatment guidelines available for long term treatment of pulmonary hypertension in LVAD patients in an ambulatory setting. We present a case of persistently high pulmonary vascular resistance in a patient with LVAD who qualified for heart transplant, after his pulmonary vascular resistance and pulmonary artery pressures were successfully reduced with combination of sildenafil, selexipag and macitentan. As to our knowledge, treatment of persistent pulmonary hypertension post LVAD with long-term selexipag has not been reported in the literature. Patients with persistent pulmonary hypertension despite LVAD implantation should be assessed and treated in the ambulatory setting with pulmonary vasodilator therapy to decrease mortality and aid in candidature for heart transplantation.
\end{abstract}

\section{Keywords}

Pulmonary vasodilator, Sildenafil, Selexipag, Macitentan, Left ventricular assist device, Pulmonary hypertension, Pulmonary vascular resistance, Cardiac transplant

\section{Introduction}

Patients with end stage heart failure often develop secondary pulmonary hypertension and subsequent- ly right ventricular (RV) failure. Despite left ventricular assist device (LVAD) implantation, some patients develop persistent pulmonary hypertension and RV failure $[1,2]$. Pulmonary hypertension, with consistently elevated pulmonary vascular resistance (PVR), is associated with poor prognosis in patients with LVAD [3] and has a linear relationship with transplant mortality [4]. Consequently, elevated PVR is a relative contraindication for orthotopic heart transplantation. Although no established medical therapy guideline has been established for chronic treatment of pulmonary hypertension in patients with LVAD, we describe the use of combining pulmonary vasodilators in ambulatory setting to reduce PVR and improve RV function in a patient with durable LVAD (Abbott Heart Mate II) which facilitated the patient's candidacy for orthotopic heart transplantation.

\section{Case}

The patient is a 69-years-old male with ischemic cardiomyopathy and left ventricular ejection fraction of $10 \%$. He initially presented to the hospital for acute on chronic systolic heart failure. Echocardiogram showed global hypokinesis and tricuspid annular plane systolic excursion of 9 millimeter. He experienced cardiac arrest early in his hospital course secondary to ventricular tachycardia (VT) with eventual return of spontaneous circulation after advanced cardiopulmonary resuscitation. An electrocardiogram exhibited acute ST depres- 
sion; coronary angiogram revealed stenosis of left anterior descending, right coronary artery (RCA) and ramus occlusions. Percutaneous coronary intervention of all three vessels was performed and an axillary percutaneous ventricular assist device (Abiomed Impella 5.0) was inserted for management of acute cardiogenic shock. Attempts were made to wean the device however the patient was device dependent and was subsequently evaluated and implanted with a durable LVAD.

Although his pulmonary capillary wedge pressure was normal, his RV was moderately dilated and hypokinetic. As a result, it became difficult to optimize his LVAD speed above 8400 revolutions per minute (RPM) as he would have persistent drop in pulsatility index despite multiple speed optimization studies with echocardiogram. Even though he was started on spironolactone $25 \mathrm{mg}$ twice daily and sildenafil $80 \mathrm{mg}$ three times a day for RV support, his LVAD speed could not be increased. The lower speed increased his risk of LVAD thrombosis, therefore a decision was made to set a higher INR goal of 2.5 and 3.5. He was discharged in stable condition 7 weeks after his day of admission.

Upon two-month follow-up, he underwent a right heart catheterization which showed elevated right atrial, RV, pulmonary artery pressures, PVR and trans-pulmonary gradient (Table 1). He was evaluated for heart transplant and was declined based on elevated PVR. In addition to the sildenafil, we started our patient on selexipag $200 \mathrm{mcg}$ twice daily and after one month his LVAD speed was increased to 8800 RPM.

Selexipag was uptitrated to $600 \mathrm{mcg}$ twice daily over 6 months. A repeat right heart catheterization showed significant improvement in right atrial, RV, pulmonary artery pressures and PVR (Table 1). Since his PVR was still above 3 woods unit, his selexipag was increased to $800 \mathrm{mcg}$. This caused him to experience side effects of facial flushing, headaches, nausea and diarrhea, there- fore the dose was decreased to $600 \mathrm{mg}$ twice daily.

Given PVR was still high to qualify for heart transplant, macitentan $10 \mathrm{mg}$ daily was started in addition to sildenafil and selexipag. A repeat echocardiogram two months after starting macitentan showed a reduction of pulmonary artery pressures. Right heart catheterization revealed PVR of less than 3 woods units on maximum tolerated dose of selexipag, macitentan and sildenafil (Table 1). We were able to set LVAD speed at 9000 RPM. He was successfully referred for heart transplantation.

\section{Discussion}

The beneficial effect of reduced PVR after LVAD implantation is not achieved in all the patients [1]. Certain patients with group 2 pulmonary hypertension develop persistently increased PVR and trans-pulmonary gradient which is typical for precapillary group 1 pulmonary hypertension. The elevated left ventricular pressure does not explain for these extremely high pulmonary resistance and pressure gradient in solo [5]. In addition, this results from a reactive increase in vascular smooth muscle tone and vascular remodeling, as chronic pulmonary congestion decreases nitric oxide and increases endothelin-1 expression [6].

The International Society for Heart and Lung Transplant (ISHLT) suggests the use of sildenafil or milrinone as an adjunct medical therapy for patients with LVAD and persistent pulmonary hypertension; however, there are no available recommendations for chronic outpatient use of pulmonary vasodilators in LVAD patients because of lack of data [7]. Studies have found sildenafil $[1,8]$ and bosentan [2] safe and well tolerated in treating pulmonary hypertension post LVAD.

The patient was particularly challenging as he had persistent pulmonary hypertension after LVAD implantation despite being on sildenafil. Although his pulmonary capillary wedge pressure was normal, due to

Table 1: Patients follow-up observation.

\begin{tabular}{|l|l|l|l|}
\hline & 2 Month Follow-up & $\mathbf{6}$ Month Follow-up & $\mathbf{1 2}$ Month Follow-up \\
\hline Vasodilator & Sildenafil & Selexipag + Sildenafil & Selexipag + Sildenafil + Macitentan \\
\hline LVAD Speed (RPM) & 8400 & 8800 & 9000 \\
\hline RAP (mmHg) & 12 & 2 & 2 \\
\hline RVP (mmHg) & $58 / 6$ & $44 / 4$ & $40 / 3$ \\
\hline PAP (mean)(mmHg) & $55 / 20(34)$ & $47 / 17(27)$ & $40 / 13(23)$ \\
\hline PCWP (mmHg) & 13 & 11 & 9 \\
\hline TPG (mmHg) & 21 & 16 & 14 \\
\hline PVR (woods units) & 5.25 & 3.98 & 2.78 \\
\hline PAPi & 2.9 & 15 & 13.5 \\
\hline CO (L/min) & 4 & 4.2 & 5.02 \\
\hline CI (L/min/m $\mathbf{2})$ & 2.6 & 2.4 & 2.97 \\
\hline
\end{tabular}

RAP: Right Atrial Pressure; RVP: Right Ventricular Pressure; PAP: Pulmonary Artery Pressure; PCWP: Pulmonary Capillary Wedge Pressure; TPG: Trans-Pulmonary Pressure; PVR: Pulmonary Vascular Resistance; PAPi: Pulmonary Artery Pulsatility Index; CO: Cardiac Output; Cl: Cardiac Index 
high PVR and worsening RV function, it was difficult to maintain high RPM of the axial flow pump. LVAD had to be set on low RPM but this increased his risk for LVAD thrombosis. To achieve a balance between acceptable RPM on LVAD and subsequently preventing LVAD thrombosis, we introduced selexipag and macitentan for RV afterload and PVR reduction. As to our knowledge, treatment of persistent pulmonary hypertension post LVAD with long-term selexipag has not been reported in the literature. This combination allowed for the VAD speed to be increased and mitigate thrombosis risk. On follow-up, the combinations of these pulmonary vasodilators (sildenafil, selexipag and macitentan) reduced PVR, and mean pulmonary artery pressure and aided our patient to qualify for orthotopic heart transplantation.

As we keenly await the results of the prospective, double-blind, randomized clinical trial SOPRANO (Clinical Study to Assess the Efficacy and Safety of Macitentan in Patients with Pulmonary Hypertension After Left Ventricular Assist Device Implantation; NCT02554930), currently, the decision to continue long-term therapy for persistent pulmonary hypertension post LVAD implantation may have to be based up on previous non-randomized studies. Pulmonary vasodilator therapy should be tailored towards individual patient after assessing the risks and benefits.

\section{Conclusion}

In the presence of persistent pulmonary hypertension despite LVAD implantation, individual patients can be assessed and treated in the ambulatory setting with pulmonary vasodilator therapy to prevent hemodynamic decline, reduce mortality and aid in candidature for heart transplantation. Given the paucity of concrete data, the selections of medications have to be individualized towards the patient.

\section{Acknowledgement}

None.

\section{Conflict of Interest}

None.

\section{Authors Contribution}

All authors have equally contributed in writing the manuscript.

\section{References}

1. Tedford RJ, Hemnes AR, Russell SD, Wittstein IS, Mahmud $\mathrm{M}$, et al. (2008) PDE5A inhibitor treatment of persistent pulmonary hypertension after mechanical circulatory support. Circ Heart Fail 1: 213-219.

2. LaRue SJ, Garcia-Cortes R, Nassif ME, Vader JM, Ray S, et al. (2015) Treatment of secondary pulmonary hypertension with bosentan after left ventricular assist device implantation. Cardiovasc Ther 33: 50-55.

3. Dang NC, Topkara VK, Mercando M, Kay J, Kruger KH, et al. (2006) Right heart failure after left ventricular assist device implantation in patients with chronic congestive heart failure. J Heart Lung Transplant 25: 1-6.

4. Hosenpud JD, Bennett LE, Keck BM, Boucek MM, Novick RJ (2000) The Registry of the International Society for Heart and Lung Transplantation: seventeenth official report-2000. J Heart Lung Transplant 19: 909-931.

5. Papathanasiou M, Ruhparwar A, Kamler M, Rassaf T, Luedike $P$ (2020) Off-label use of pulmonary vasodilators after left ventricular assist device implantation: Calling in the evidence. Pharmacol Ther 214: 214107619.

6. Delgado JF, Conde E, Sánchez V, López-Ríos F, Gómez-Sánchez MA, et al. (2005) Pulmonary vascular remodeling in pulmonary hypertension due to chronic heart failure. Eur J Heart Fail 7: 1011-1016.

7. Mehra MR, Canter CE, Hannan MM, Semigran MJ, Uber PA, et al. (2016) The 2016 International Society for Heart Lung Transplantation listing criteria for heart transplantation: A 10-year update. J Heart Lung Transplant 35: 1-23.

8. Baker WL, Radojevic J, Gluck JA (2016) Systematic Review of Phosphodiesterase-5 Inhibitor Use in Right Ventricular Failure Following Left Ventricular Assist Device Implantation. Artif Organs 40: 123-128. 